\title{
Bivariate longitudinal data analysis: a case of hypertensive patients at Felege Hiwot Referral Hospital, Bahir Dar, Ethiopia
}

\author{
Demeke Lakew Workie*, Dereje Tesfaye Zike and Haile Mekonnen Fenta
}

\begin{abstract}
Objective: Longitudinal data are often collected to study the evolution of biomedical markers. The study of the joint evolution of response variables concerning hypertension over time was the aim of this paper. A hospital based retrospective data were collected from September 2014 to August 2015 to identify factors that affect hypertensive. The joint mixed effect model with unstructured covariance was fitted. A total of 172 patients screened for antihypertensive drugs treated were longitudinally considered from Felege Hiwot referral.

Results: The joint mixed effect model with unstructured covariance (AIC: $12,236.9$ with $\chi_{12}^{2}=1007.8, P<10^{-4}$ ) was significantly best fit to the data. The correlation between the evolutions of DBP and SBP was 0.429 and the evolution of the association between responses over-time was found 0.257. Among all covariates included in joint-mixedeffect-models, sex, residence, related disease and time were statistically significant on evolution of systolic and diastolic blood pressure. The joint modeling of longitudinal bivariate responses is necessary to explore the association between paired response variables like systolic and diastolic blood pressure. Fitting joint model with modern computing method is recommended to address questions for association of the evolutions with better accuracy.
\end{abstract}

Keywords: Joint mixed effect models, Systolic blood pressure, Diastolic blood pressure hypertension

\section{Introduction}

Hypertension is a chronic condition resulting from high blood pressure in the arteries during circulation. Clinically, a person is said to be hypertensive if the individual's systolic blood pressure (SBP) is greater than $140 \mathrm{~mm}$ $\mathrm{Hg}$ and or diastolic blood pressure (DBP) is greater than $90 \mathrm{mmHg}$ and those who were already under medication $[1,2]$. Monitoring hypertension is a necessity for patients to assess the progression of blood pressure. Typically two markers (systolic and diastolic blood pressure, SBP and DBP) are measured repeatedly over time after treatment to ensure that no signs of blood pressure problems. These markers are needed to ensure an accurate evaluation of the blood pressure since they are correlated and could be influenced by the socioeconomic status of the patient [3]. Given the interdependence of these outcomes

*Correspondence: demay_gu06@yahoo.com Department of Statistics, Bahir Dar University, Peda Campus, P.O.Box: 79, Bahir Dar, Ethiopia in determining blood pressure, it is important to evaluate the factors that affect the rate of change in these outcomes in a joint manner [4].

When several markers are measured repeatedly, joint modeling was used in the context of jointly studying time to clinical event and repeated measures on surrogate outcomes [5-8]. Others included joint modeling of the multilevel item response theory (MLIRT) and Cox's proportional hazard model for time to dependent terminal event with shared random effects to link the two models $[9,10]$. As Jaffa et al. [5], Thiebaut et al. [11] and Fiewus and Verebeke [12-14] stated a joint modeling of biomarker data is necessary to quantify, the relationship between evolutions of different responses and the evolution of the relationship between different response variables over time. Thus, the aim of this study was using the joint mixed effect model with unstructured covariance structure to answer the evolution of systolic and diastolic blood pressure over time. 


\section{Main text Methods}

Hospital based retrospective studies were conducted among hypertensive patients attending antihypertensive clinic between September 2014 to August 2015 at Felege Hiwot referral hospital, Bahir Dar, Ethiopia. The area is selected because it is central referral hospital that provides organized hypertension follows up care. The sample size was calculated using 95\% confidence level and the overall sample size was 172 which after adding $5 \%$ for non-response. A systematic random sampling method was adopted for selecting a representative sample from the list of the medical charts that contain the list of hypertensive patients' name and identification number. Patients were selected randomly using their unique identification number. The study considered all hypertensive patients, whose age was above 15 years regardless of their treatment category during the study period in the referral hospital.

In this paper, a longitudinal data on two markers of hypertension (SBP and DBP), various socioeconomic status covariates and clinical factors were considered. SBP and DBP measurements were collected at baseline (SBP0 and DBP0), and every 3 months (SBP3 to SBP12 and DBP3 to DBP12) thereafter. In order to ensure sufficient available information, only a sub-sample of patients having at least four repeated with equally spaced measurements were included in the analyses. Thus, a follow-up data were included hypertensive patients had a maximum of five repeated measures on blood pressure. Data were entered and edited in SPSS v. 21 and analyzed by SAS v. 9.4 and the $95 \%$ confidence interval was used to determine significance tests. The missing data for both markers were replaced by last observation carried forward (LOCF) as [15].

\section{Bivariate random mixed effect model}

Multivariate longitudinal data arise when a set of different responses on the same unit are measured repeatedly over time. A joint modeling of such kind of data is necessary to quantify, firstly, the relationship between evolutions of different responses and, secondly, the evolution of the relationship between different response variables over time. Thus, a pair wise fitting approach has been used in this study as proposed in the literature $[12,16]$. The joint modeling approach investigated in this study was the bivariate longitudinal mixed effect models that included both fixed and random effects. Thus, in this study $\mathrm{DBP}_{\mathrm{ij} 1}$ and $\mathrm{SBP}_{\mathrm{ij} 2}$ be the bivariate outcomes for the ith subject measured at jth times for outcomes 1 and 2 .

We define a general bivariate linear mixed effect model including a random component, a variance-covariance process, and an independent error. Let $Y_{i}^{k}=\left[\begin{array}{c}D B P_{i} \\ S B P_{i}\end{array}\right]$, the response vector for the ith subject with $D B P_{i}$ and $S B P_{i}$ having $\mathrm{n}_{\mathrm{i}}$ sampled measurements of the marker $\mathrm{k}(\mathrm{k}=1$, 2).

To take into account correlation between both markers the following bivariate linear mixed effect model was used.

The mixed-effect models assume that the regression coefficients are a random sample from some population of the possible coefficient and allow one to model variations between study units [17]. The random Coefficient mixed model gives that two random slopes (one for DBP and one for SBP) be fitted for each individual and the variances of measurement errors are different for different markers. The covariance matrix for the random slopes is $G=\left[\begin{array}{cc}\sigma_{1}^{2} & \sigma_{12}^{2} \\ \sigma_{21}^{2} & \sigma_{2}^{2}\end{array}\right]$, and the two markers are independent of $G=\left[\begin{array}{cc}\sigma_{1}^{2} & 0 \\ 0 & \sigma_{2}^{2}\end{array}\right]$

In this paper, a bivariate linear mixed model including random effects and independent measurement error for both SBP and DBP was presented as outcome variables in each occasion. The two end points were longitudinally measured as a vector of responses, $\mathrm{Y}_{\mathrm{i}}$, at each occasion with this model: $Y_{i}=X_{i} \beta+Z_{i} \gamma_{i}+W_{i}+\varepsilon_{i}$

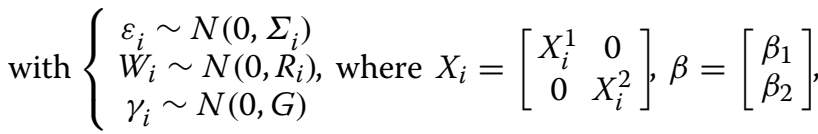
$Z_{i}=\left[\begin{array}{cc}Z_{i}^{1} & 0 \\ 0 & Z_{i}^{2}\end{array}\right], \gamma_{i}=\left[\begin{array}{l}\gamma_{i}^{1} \\ \gamma_{i}^{2}\end{array}\right]$ and $W_{i}=\left[\begin{array}{c}W_{i}^{1} \\ W_{i}^{2}\end{array}\right]$ is a $2 n_{i^{-}}$ vector of realization of a variance-covariance process $w_{i}(t)=\left[\begin{array}{c}w_{i}^{1}(t) \\ w_{i}^{2}(t)\end{array}\right]$ and $\varepsilon_{i}=\left[\begin{array}{c}\varepsilon_{i}^{1} \\ \varepsilon_{i}^{2}\end{array}\right]$ represents independent measurement errors. Though many socioeconomic covariates and clinical factors were considered in the analysis, only covariates significantly associated with systolic and diastolic blood pressure were reported.

\section{Results}

The sample was composed of 172 patients with antihypertensive drugs treated, of which 38 (22.09\%) and 21 (12.51\%) patients had diabetic and stroke and 76 (44.19\%) patients had no other related disease. The results showed that, $93(54.09 \%)$ women, with a mean age of 51.87 $(\mathrm{SD}=14.33)$ years. Whereas mean age of male patients was $56.82(\mathrm{SD}=14.98)$ years and the mean systolic and diastolic blood pressure of patients declined from baseline to the next 3 months period, to the next 6 months period and soon up to the last 12 months period follow 
Table 1 Patients' demographic characteristics

\begin{tabular}{lccll}
\hline Variables & $\mathbf{n}(\%)$ & Mean (SD) & Min-max & $\mathbf{9 5 \%} \mathbf{C l}$ \\
\hline Sex & & & & \\
Male & $79(45.93)$ & & & \\
Female & $93(54.07)$ & & & \\
Residence & & & & \\
Urban & $101(59.06)$ & & & \\
Rural & $70(40.94)$ & & & \\
Other related disease & & & \\
None & $76(44.19)$ & & & \\
Others & $37(21.51)$ & & & \\
Stroke & $21(12.51)$ & & & \\
Diabetic & $38(22.09)$ & & & \\
Age in years & & & & \\
Male & 79 & $56.82(14.49)$ & $20.00-86.00$ & $53.58-60.07$ \\
Female & 92 & $51.87(14.33)$ & $25.00-92.00$ & $48.90-54.84$ \\
Total & 171 & $54.16(14.57)$ & $20.00-92.00$ & $51.96-56.36$ \\
SBP in mmHg & & & & \\
SBP0 & 172 & $153.58(31.60)$ & $60.00-240.00$ & $148.82-158.33$ \\
SBP1 & 172 & $142.47(23.33)$ & $80.00-210.00$ & $138.96-145.98$ \\
SBP2 & 172 & $135.78(22.99)$ & $80.00-220.00$ & $132.33-139.24$ \\
SBP3 & 172 & $133.84(23.18)$ & $80.00-240.00$ & $130.35-137.33$ \\
SBP4 & 172 & $129.48(20.07)$ & $80.00-200.00$ & $126.46-132.50$ \\
DBP in mmHg & & & \\
DBP0 & 172 & $94.22(17.80)$ & $50.00-160.00$ & $91.54-96.89$ \\
DBP1 & 172 & $86.54(15.30)$ & $40.00-140.00$ & $84.24-88.84$ \\
DBP2 & 172 & $82.96(14.36)$ & $20.00-140.00$ & $80.80-85.12$ \\
DBP3 & 172 & $82.67(13.33)$ & $50.00-130.00$ & $80.67-84.68$ \\
DBP4 & 172 & $83.02(13.25)$ & $50.00-170.00$ & $81.03-85.02$ \\
\hline
\end{tabular}

$S D$ standard deviation, Min-Max minimum-maximum, $\mathrm{Cl}$ confidence interval, $S B P$ systolic blood pressure, $D B P$ diastolic blood pressure, $\mathrm{mmHg}$ millimeter mercury

up time. The baseline SBP mean of patients was 153.58 $(\mathrm{SD}=31.60) \mathrm{mmHg}$ and declined to $129.48(\mathrm{SD}=20.07)$ $\mathrm{mmHg}$ over time and similar history was found in DBO patients. These summaries suggest that the treatment has a significant effect on systolic and diastolic blood pressure over the follow-up time (Table 1).
Figure 1 shows mean estimates with 95\% CI, for the SBP and DBP separately by different factors. Clearly, observe that decreasing trend in both SBP and DBP over time. The mean estimate of blood pressure for a female was more than male at baseline and significantly different $(\mathrm{P}<0.0001)$ between the outcomes. Whereas, the mean estimate of diastolic and systolic blood pressure for rural and urban area seems to no difference The bivariate random mixed-effects model was fitted to show the association of correlate measurements to the 3-month-interval for blood pressure to identify the risk factors.

The bivariate random mixed-effects model was significantly best with AIC value of 12,236.9 and $\chi_{12}^{2}=1007.8$, $\mathrm{P}<10^{-4}$. Output obtained by the bivariate random mixed effects model with UN provides estimations for the covariance matrix and fixed effects. The variance-covariance matrix for the random effects and the error components for each response were obtained as:

$$
\begin{aligned}
& G=\left[\begin{array}{cccc}
\sigma_{a_{1}}^{2} & \sigma_{a_{1} b_{1}} & \sigma_{a_{1} a_{2}} & \sigma_{a_{1} b_{2}} \\
& \sigma_{b_{1}}^{2} & \sigma_{b_{1} a_{2}} & \sigma_{b_{1} b_{2}} \\
& & \sigma_{a_{2}}^{2} & \sigma_{a_{2} b_{2}} \\
& & & \sigma_{b_{2}}^{2}
\end{array}\right] \\
& =\left[\begin{array}{rrrr}
153.21 & 169.80 & 76.13 & 168.90 \\
& 402.77 & 116.83 & 435.72 \\
& & 219.62 & 171.44 \\
& & & 725.77
\end{array}\right] \text { and } \\
& \mathrm{R}=\left[\begin{array}{c}
\sigma_{1}^{2} \sigma_{12} \\
\sigma_{2}^{2}
\end{array}\right]=\left[\begin{array}{cc}
58.76 & 90.61 \\
& 194.55
\end{array}\right]
\end{aligned}
$$

The correlation between the evolutions for the two random slopes is given by:

$$
\begin{aligned}
r_{E} & =\frac{\operatorname{Cov}\left(b_{1}, b_{2}\right)}{\sqrt{\operatorname{Var}\left(b_{1}\right) * \operatorname{Var}\left(b_{2}\right)}}=\frac{\sigma_{b_{1} b_{2}}}{\sqrt{\sigma_{b_{1}}^{2} * \sigma_{b_{2}}^{2}}} \\
& =\frac{171.44}{\sqrt{219.62 * 725.77}}=0.429
\end{aligned}
$$

whereas, the marginal correlation between the two responses as a function of time is given by:

$$
\begin{aligned}
\mathrm{r}_{\mathrm{m}}(\mathrm{t})= & \frac{\sigma_{a_{1} a_{2}}+t\left(\sigma_{a_{1} b_{2}}+\sigma_{b_{1} a_{2}}\right)+t^{2} \sigma_{b_{1} b_{2}}}{\sqrt{\left(\sigma_{a_{1}}^{2}+2 t^{2}\left(\sigma_{a_{1} b_{1}}+\sigma_{b_{1}}^{2}\right)+\sigma_{1}^{2}\right) *\left(\sigma_{a_{2}}^{2}+2 t^{2}\left(\sigma_{a_{2} b_{2}}+\sigma_{b_{2}}^{2}\right)+\sigma_{2}^{2}\right)}} \\
& \times \frac{76.13+t(168.9+116.83)+t^{2} 435.72}{\sqrt{\left(153.21+2 t^{2}[169.8+402.77]+58.76\right) *\left(219.62+2 t^{2}[171.44+725.77]+194.55\right)}} \\
= & \frac{435.72 t^{2}+285.73 t+76.13}{\sqrt{\left(211.97+1145.4 t^{2}\right) *\left(414.17+1794.42 t^{2}\right)}}=\left[\begin{array}{c}
0.257 \\
0.368 \\
0.337 \\
0.327 \\
0.320
\end{array}\right]
\end{aligned}
$$



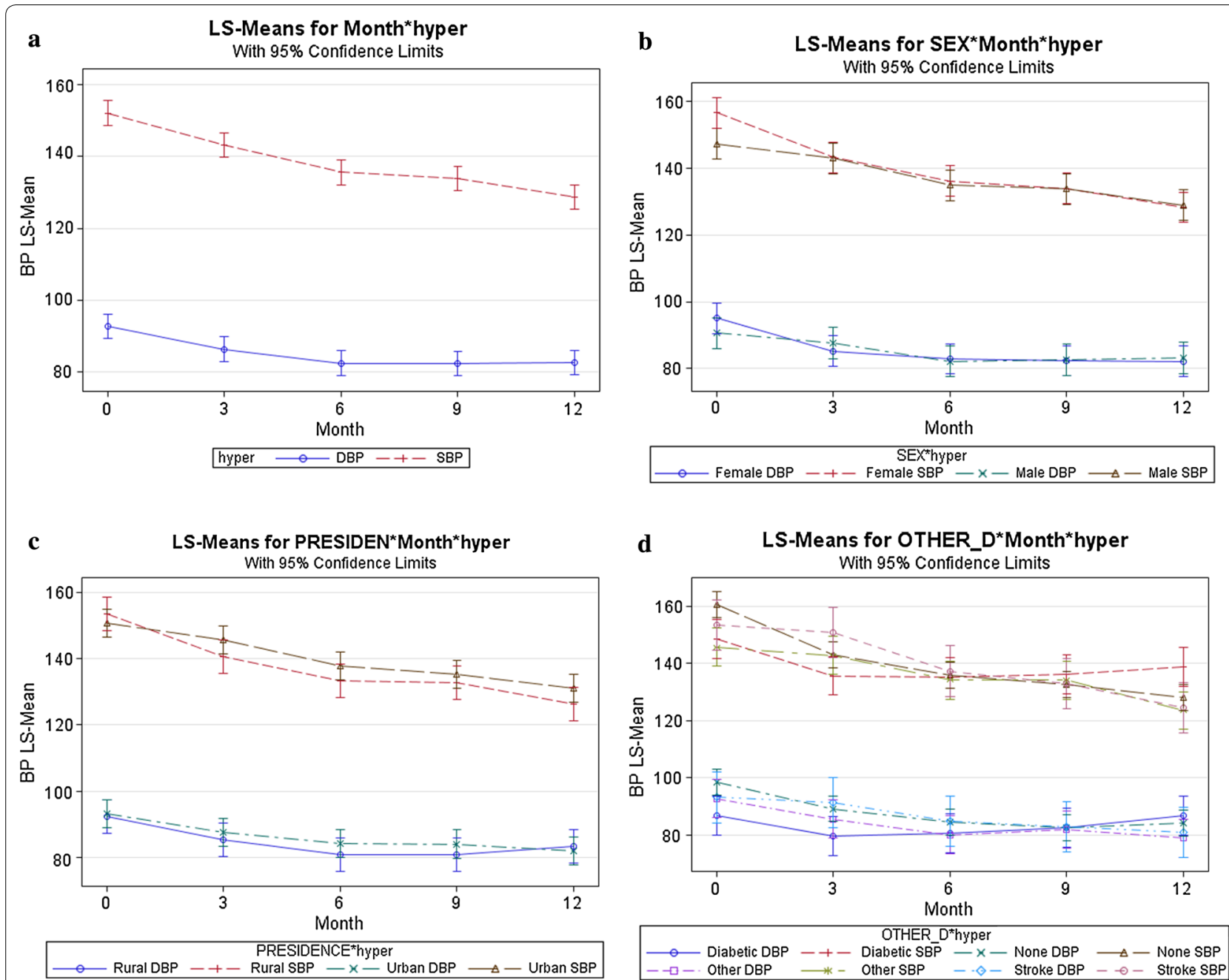

Fig. 1 Mean estimates with $95 \% \mathrm{Cl}$ a Systolic and diastolic BP. b Systolic and diastolic BP by sex. c Systolic and diastolic BP by place of residence. d Systolic and diastolic BP by related disease

The result revealed that the correlation between the diastolic and systolic blood pressure for random intercepts at baseline was 0.257 and the remaining marginal correlation for 3 to 12 months were $0.368,0.337,0.327$ and 0.320 , respectively over the 3 month period follow up time.

The primary focus of this study was interested in modeling longitudinal measures of SBP and DBP patients as a function of baseline age, sex, residence and related disease. The bivariate random mixed-effects model revealed that baseline $(P=0.0001), 3$ months period $(P=0.001)$ and 6 months period $(\mathrm{P}=0.011)$ systolic and diastolic blood pressure were significantly different compared with 12 months period follow up time. The systolic and diastolic blood pressure of rural patients $(\mathrm{P}=0.0308)$ was significantly different compared with urban patients.
The systolic and diastolic blood pressure patients with diabetic showed a significant difference at baseline $(P=0.0077)$ and 3 months period $(P=0.0015)$ compared to patients with stroke (Table 2).

\section{Discussion}

The joint mixed effect model with unstructured covariance (AIC 12,236.9 with $\chi_{12}^{2}=1007.8, \mathrm{P}<10^{-4}$ ) was significantly best fit to the covariates. This results in line with $[18,19]$ as they used a joint model for a longitudinal data and obtained the best final model with UN compared to others.

The correlation between the evolutions for the DBP and SBP was 0.429. This result was in line with a joint model fitted for female SBP and DBP that considered the before and after drug administration data [20]. Similarly, 
Table 2 Solution for fixed effects

\begin{tabular}{|c|c|c|c|c|c|c|}
\hline Effect & Category & Estimate & Standard error & DF & $\mathrm{t}$ value & $\operatorname{Pr}>|t|$ \\
\hline Age & & -0.07951 & 0.04934 & 1341 & -1.61 & 0.1073 \\
\hline Sex $($ male $=$ ref $)$ & Female & 130.26 & 4.6367 & 1341 & 28.09 & $<.0001$ \\
\hline Residence (urban = ref) & Rural & 2.6875 & 2.2787 & 1341 & 1.18 & 0.2385 \\
\hline \multirow[t]{3}{*}{ Other disease (stroke $=$ ref) } & Diabetic & 4.4679 & 4.0411 & 1341 & 1.11 & 0.2691 \\
\hline & None & 2.4592 & 3.6329 & 1341 & 0.68 & 0.4986 \\
\hline & Other & -1.8215 & 4.0377 & 1341 & -0.45 & 0.6520 \\
\hline \multirow[t]{4}{*}{ Month (after 12 months = ref) } & $0=$ Baseline & 25.4167 & 4.7397 & 1341 & 5.36 & $<.0001$ \\
\hline & $3=$ After 3 months & 22.7743 & 4.7397 & 1341 & 4.81 & $<.0001$ \\
\hline & $6=$ After 6 months & 12.0668 & 4.7397 & 1341 & 2.55 & 0.0110 \\
\hline & $9=$ After 9 months & 8.2842 & 4.7397 & 1341 & 1.75 & 0.0807 \\
\hline \multirow[t]{4}{*}{ Sex*month (male ${ }^{*} 12=$ ref $)$} & Female*0 & 3.7734 & 2.7517 & 1341 & 1.37 & 0.1705 \\
\hline & Female*3 & -1.9921 & 2.7517 & 1341 & -0.72 & 0.4692 \\
\hline & Female*6 & 0.8489 & 2.7517 & 1341 & 0.31 & 0.7577 \\
\hline & Female*9 & 0.1634 & 2.7517 & 1341 & 0.06 & 0.9527 \\
\hline \multirow[t]{4}{*}{ Residence*month (urban*12 = ref) } & Rural*0 & -3.5497 & 2.8104 & 1341 & -1.26 & 0.2068 \\
\hline & Rural*3 & -4.3474 & 2.8104 & 1341 & -1.55 & 0.1221 \\
\hline & Rural*6 & -6.0771 & 2.8104 & 1341 & -2.16 & 0.0308 \\
\hline & Rural*9 & -5.0831 & 2.8104 & 1341 & -1.81 & 0.0707 \\
\hline \multirow[t]{12}{*}{ Other disease ${ }^{*}$ month (stroke ${ }^{*} 12=$ ref) } & Diabetic*0 & -13.2490 & 4.9672 & 1341 & -2.67 & 0.0077 \\
\hline & Diabetic*3 & -17.3071 & 4.9672 & 1341 & -3.48 & 0.0005 \\
\hline & Diabetic*6 & -9.8464 & 4.9672 & 1341 & -1.98 & 0.0477 \\
\hline & Diabetic*9 & -5.5524 & 4.9672 & 1341 & -1.12 & 0.2638 \\
\hline & None $^{*} 0$ & 1.1267 & 4.4649 & 1341 & 0.25 & 0.8008 \\
\hline & None*3 & -4.9565 & 4.4649 & 1341 & -1.11 & 0.2672 \\
\hline & None*6 & -3.0551 & 4.4649 & 1341 & -0.68 & 0.4939 \\
\hline & None*9 & -2.6169 & 4.4649 & 1341 & -0.59 & 0.5579 \\
\hline & Other*0 & 1.2464 & 4.9616 & 1341 & 0.25 & 0.8017 \\
\hline & Other*3 & -4.0513 & 4.9616 & 1341 & -0.82 & 0.4143 \\
\hline & Other*6 & -2.7634 & 4.9616 & 1341 & -0.56 & 0.5777 \\
\hline & Other*9 & 0.3122 & 4.9616 & 1341 & 0.06 & 0.9498 \\
\hline
\end{tabular}

DF degree of freedom, ref reference category

the evolution of the association between the responses over time was found 0.257 . The remaining marginal correlation between DBP and SBP was 0.368, 0.337, 0.327 and 0.320 , respectively over the follow-up time. As the marginal correlation converges to the random slopes, the relationship was underlined by the correlation between DBP and SBP at each follow-up time [12].

The mean difference in systolic and diastolic blood pressure for male and female had only significant $(\mathrm{P}<0.034)$ between the baseline and after a 12-month follow-up-time. This result in line with [21-23] as they stated hypertension is the leading cause of death in women than that of men and they concluded that antihypertensive treatment decreases the death of women. The current study revealed that there is an association between blood pressure at baseline and during follow-up among patients with stroke and diabetes. This result was similar to the study in the Louisiana State University Hospital-based longitudinal data [24-26], and blood pressure at baseline and during follow-up and the risk of all-cause mortality among patients with diabetes [27].

\section{Conclusion and recommendation}

The joint mixed effect model with unstructured covariance was preferred among others to fit the data. It can be generalized that, the two outcomes have a strong positive correlation and the joint mixed effect model was preferred. Thus, joint modeling of longitudinal bivariate responses is necessary to explore the association between paired response variables. The baseline mean of the two symptoms was out of the normal range for hypertensive patients but it declines through clinical treatment 
follow-up time of 3 month period intervals. As the joint model is able to address questions for the association of the evolutions with better accuracy, fitting joint mixed effect model is recommended.

\section{Limitations}

The joint modeling problem is failing to convergence because of a large number of parameter estimates. Moreover, some one also might want to look at modeling more than two response variables over time. Thus one might be able to be implemented using modern computing method for future work to model more than two response variables over time. Here in the joint mixed effect model, authors did not see the interaction effect of the predictors over time. Therefore, researchers should consider the contribution of the interaction effect of the predictors on to the joint mixed effect model.

\section{Abbreviations}

AIC: Akaike Information Criterion; Cl: confidence interval; DBP: diastolic blood pressure; LOCF: last observation carried forward; MLIRT: multilevel item response theory; SAS: statistical analysis system; SBP: systolic blood pressure; SD: standard deviation; SPSS: Statistical package for Social Science; UN: unstructured.

\section{Authors' contributions}

$\mathrm{DL}$ initiated the research and involved in the write up of the proposal, the data entry and data analysis, and write up of the manuscript. DT and HM initiated the research, wrote the research proposal, conducted the research, did data entry and analysis and wrote the manuscript. All authors read and approved the final manuscript.

\section{Acknowledgements}

The authors wish to thank the study participants and data collectors for making this study possible.

\section{Competing interests}

The authors declared that they have no competing interests.

\section{Availability of data and materials}

If needed the raw data in SPSS and SAS code for this article is available.

\section{Consent for publication}

Not applicable.

\section{Ethics approval and consent to participate}

This study was reviewed and approved by the Bahir Dar University of Science College Research Ethics Committee and Felege Hiwot Referal Hospital Patient's Ethics Committee. As the study was based on retrospective data, informed consent was not needed.

\section{Funding}

This research was funded by Science College Research and Community Service, Bahir Dar University.

\section{Publisher's Note}

Springer Nature remains neutral with regard to jurisdictional claims in published maps and institutional affiliations.

Received: 23 June 2017 Accepted: 30 November 2017

Published online: 08 December 2017
References

1. World Health Organization. Global status report on noncommunicable diseases 2010, WHO library cataloguing-in-publication data. Geneva: World Health Organization; 2011. ISBN 978-92-4-156422-9.

2. Choi YH, Chowdhury R, Swaminathan B. Prediction of hypertension based on the genetic analysis of longitudinal phenotypes: a comparison of different modeling approaches for the binary trait of hypertension. BMC Proc. 2014;8(Suppl 1):s78.

3. James GD, Sealey JE, Alderman M, Ljungman S, Mueller FB, Pecker MS, et al. A longitudinal study of urinary creatinine and creatinine clearance in normal subjects race, sex, and age differences. Am J Hypertens. 1988;1(2):124-31.

4. Beunckens C, Molenberghs G, Thijs H, Verbeke G. Incomplete hierarchical data. Stat Methods Med Res. 2007;16(5):457-92.

5. Jaffa MA, Gebregziabher M, Jaffa AA. Analysis of multivariate longitudinal kidney function outcomes using generalized linear mixed models. J Transl Med. 2015;13(1):192.

6. Xu J, Zeger SL. The evaluation of multiple surrogate endpoints. Biometrics. 2001;57(1):81-7.

7. Brown ER, Ibrahim JG, DeGruttola V. A flexible B-spline model for multiple longitudinal biomarkers and survival. Biometrics. 2005;61(1):64-73.

8. Brown ER, Ibrahim JG. Bayesian approaches to joint cure-rate and longitudinal models with applications to cancer vaccine trials. Biometrics. 2003;59(3):686-93.

9. He B, Luo S. Joint modeling of multivariate longitudinal measurements and survival data with applications to Parkinson's disease. Stat Methods Med Res. 2016;25(4):1346-58.

10. Twisk JW. Applied longitudinal data analysis for epidemiology: a practical guide. Cambridge: Cambridge University Press; 2013.

11. Thiébaut R, Jacamin-Gadda H, Chêne G, Leport C, Commenges D. Bivariate linear mixed models using SAS proc MIXED. Comput Methods Programs Biomed. 2002;69(3):249-56.

12. Fieuws $S$, Verbeke $G$. Joint modeling of multivariate longitudinal profiles: pitfalls of the random-effects approach. Stat Med. 2004;23(20):3093-104.

13. Chakraborty H, Helms RW, Sen PK, Cohen MS. Estimating correlation by using a general linear mixed model: evaluation of the relationship between the concentration of HIV-1 RNA in blood and semen. Stat Med. 2003;22(9):1457-64.

14. Bahçecitapar M, Karadag Ö, Aktas S. Specification of variance-covariance structure in bivariate mixed model for unequally time-spaced longitudinal data. Int J Stat Med Res. 2015;4(4):370.

15. Verbeke $\mathrm{G}$. Linear mixed models for longitudinal data. Linear mixed models in practice. Newyork: Springer; 1997. p. 63-153.

16. Fieuws $S$, Verbeke G. Pairwise fitting of mixed models for the joint modeling of multivariate longitudinal profiles. Biometrics. 2006;62(2):424-31.

17. Littell RC, Milliken GA, Stroup WW, Wolfinger RD, Schabenberger O. SAS for mixed models. Cary: SAS Institute; 2007.

18. Fissuh $\mathrm{YH}$, Muletav G. A joint model for a longitudinal pulse rate and respiratory rate of congestive heart failure patients: at Ayder Referral Hospital of Mekelle University, Tigray, Ethiopia. J Biom Biostat. 2015;6(5):1.

19. Facts HA. State heart disease and stroke prevention programs addresses heart attack prevention. 2006.

20. Lambert P, Vandenhende F. A copula-based model for multivariate nonnormal longitudinal data: analysis of a dose titration safety study on a new antidepressant. Stat Med. 2002;21(21):3197-217.

21. Ong KL, Tso AW, Lam KS, Cheung BM. Gender difference in blood pressure control and cardiovascular risk factors in Americans with diagnosed hypertension. Hypertension. 2008;51(4):1142-8.

22. Gu Q, Burt VL, Paulose-Ram R, Dillon CF. Gender differences in hypertension treatment, drug utilization patterns, and blood pressure control among US adults with hypertension: data from the National Health and Nutrition Examination Survey 1999-2004. Am J Hypertens. 2008;21(7):789-98.

23. Keyhani S, Scobie JV, Hebert PL, McLaughlin MA. Gender disparities in blood pressure control and cardiovascular care in a national sample of ambulatory care visits. Hypertension. 2008;51(4):1149-55.

24. Zhao W, Katzmarzyk PT, Horswell R, Wang Y, Johnson J, Cefalu WT, et al. Blood pressure and stroke risk among diabetic patients. J Clin Endocrinol Metab. 2013;98(9):3653-62. 
25. Li W, Wang Y, Chen L, Horswell R, Xiao K, Besse J, et al. Increasing prevalence of diabetes in middle or low-income residents in Louisiana from 2000 to 2009. Diabetes Res Clin Pract. 2011;94(2):262-8.

26. Zhang Y, Li W, Wang Y, Chen L, Horswell R, Xiao K, et al. Increasing prevalence of hypertension in low-income residents within Louisiana state university health care services division hospital system. Eur J intern Med. 2012;23(8):e179-84

27. Li W, Katzmarzyk PT, Horswell R, Wang Y, Johnson J, Hu G. Blood pressure and all-cause mortality among patients with type 2 diabetes. Int J Cardiol. 2016;1:206 (Epub 121).

\section{Submit your next manuscript to BioMed Central and we will help you at every step:}

- We accept pre-submission inquiries

- Our selector tool helps you to find the most relevant journal

- We provide round the clock customer support

- Convenient online submission

- Thorough peer review

- Inclusion in PubMed and all major indexing services

- Maximum visibility for your research

Submit your manuscript at www.biomedcentral com/submit
() Biomed Central 